\title{
Health vigilance concerning bacteremia in neonatology - neonatal intensive care unit at the Mohammed VI University Hospital of Oujda (Morocco): epidemiological profile and antibiotic resistance
}

\author{
Loubna Yacoubi ${ }^{1,}{ }^{*}$, Soumia Farih ${ }^{1}$, Abderazzak Seddari ${ }^{1}$, Noussaiba Benhamza ${ }^{1}$, Adnane Aarab ${ }^{1}$, and Adil maleb ${ }^{1}$ \\ ${ }^{1}$ Microbiology Laboratory. Mohammed VI University Hospital of Oujda (Morocco), Medicine and Pharmacy of Oujda, Mohammed I \\ University, Morocco.
}

\begin{abstract}
The objective of our study was to describe the epidemiological profile of blood culture isolates in the neonatology - neonatal intensive care unit of the Mohammed VI University Hospital of Oujda (Morocco) and to specify the resistance profile of the main germs isolated to antibiotics. This is a retrospective and descriptive study of 21 months from December 14, 2016 to September 14, 2018 and covering all positive blood cultures processed in the microbiology laboratory in accordance with REMIC (reference in medical microbiology) and EUCAST (European Committee on Antimicrobial Susceptibility Testing).contaminated blood cultures were excluded. As results, we collected 275 positive blood cultures. They occurred in the context of intravascular device (IVD) use in 59\% ( $\mathrm{n}=162)$ of cases. The most isolated bacterial groups were coagulase-negative staphylococci (CNS) $41.45 \%(\mathrm{n}=114)$, followed by Enterobacteriaceae $32.36 \%($ $\mathrm{n}=89)$.CNS were resistant to all beta-lactams in $57.89 \%(\mathrm{n}=66)$, and to glycopeptides in $5.26 \%(\mathrm{n}=6)$. Enterobacteriaceae were producers of extended-spectrum betalactamases in $79.77 \%(n=71)$ and producers of carbapenemases in $13.48 \%(\mathrm{n}=12)$ of cases. The alarming increase of enterobacteriaceae isolates and their antibiotic resistance rates should encourage the reinforcement of hygiene measures in our University Hospital.
\end{abstract}

\section{Introduction}

Incomplete inflammatory immune responses make newborns more susceptible to bacterial invasion of the bloodstream, and the risk is even higher in children born prematurely [ 1]

With improved obstetrical management and the use of antibiotics, early neonatal sepsis is becoming less common. However, it is one of the most frequent causes of neonatal morbidity and mortality in the preterm population. [2]

All newborns with suspected sepsis are evaluated promptly and started on antibiotic therapy before bacteriological confirmation. Early identification of an organism and appropriate antibiotic treatment are essential to prevent increasing mortality and morbidity [3]

The objective of our study was to describe the epidemiological profile of blood culture isolates in the neonatology-neonatal intensive care unit of the
Mohammed VI University Hospital of Oujda (Morocco) and to specify the resistance profile of the main germs isolated to antibiotics

\section{Materials and methods}

This was a 21-month retrospective study from December 14, 2016 to September 14, 2018, of all positive blood cultures processed at the Microbiology Laboratory. Contaminated blood cultures were excluded

Blood cultures were performed by peripheral venipuncture or femoral vein and sometimes at the central venous catheter (CVC). A quantity of blood (depending on the weight) is taken and injected into a pediatric blood culture vial (BD Bactec Ped plus vials). After verification of non-compliance, the vials are recorded and incubated in the BD FX 400 automated system for a maximum of 7 days (beyond this period, the microorganisms detected are generally contaminants).

\footnotetext{
*Loubna Yacoubi : loubnayacoubi1993@gmail.com
} 
The vials detected positive by the BD BACTEC are discharged from the automaton and analyzed. The culture was performed on enriched and selective agar media and incubated at $37^{\circ} \mathrm{C}$ for 24 to 48 hours.

Identification at the laboratory level was based on morphological, cultural, biochemical and conventional antigenic characteristics. Biochemical identification was done by Api galleries, determined with the BD Phoenix 100

Antibiotic susceptibility testing was performed in accordance with the recommendations of the Antibiotic susceptibility Committee of the French Society of Microbiology (CA-SFM) and the European Committee on Antimicrobial Susceptibility Testing (EUCAST) [4]. The results were interpreted in accordance with the recommendations of the medical microbiology reference system (REMIC) [5].

\section{Results:}

\section{Microbiological epidemiology}

During the study period, we collected 275 positive blood cultures. They occurred in the context of intravascular device (IVD) use in 59\% $(n=162)$ of cases. The most isolated bacterial groups were coagulase-negative staphylococci $\quad(n=114 ; 41.45 \%)$,followed by enterobacteria $(n=89 ; 32.36 \%)$, including $20.36 \%(n=56)$ of Klebsiella pneumoniae; then Acinetobacter Baumannii in $10.9 \%(n=30)$. (Table 1$)$

Table 1: Distribution according to the germs isolated

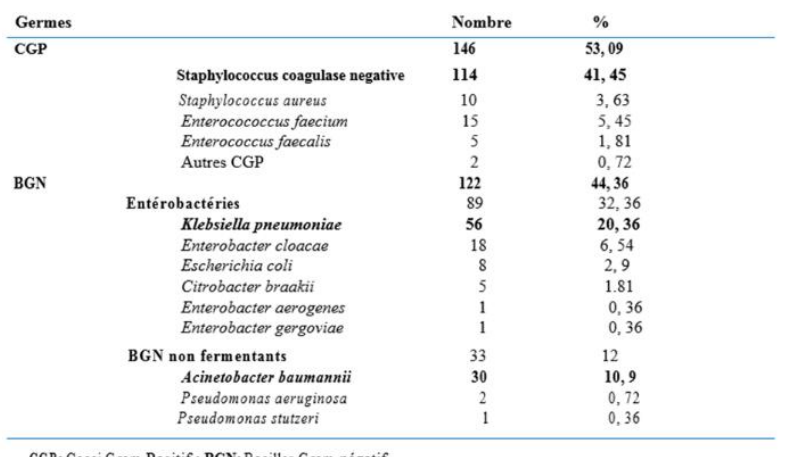

\section{Resistance profile}

CNS were resistant to all beta-lactams in $57.89 \%(n=66)$, and to glycopeptides in $5.26 \%(n=6)$. Enterobacteriaceae were producers of extended-spectrum betalactamases in $79.77 \%(\mathrm{n}=71)$ of cases and carbapenemases in $13.48 \%$ $(\mathrm{n}=12)$ of cases. $96 \%$ of Klebsiella pneumoniae strains were ESBL producers; $73 \%(n=22)$ of Acinetobacter baumannii strains were carbapenemase producers.

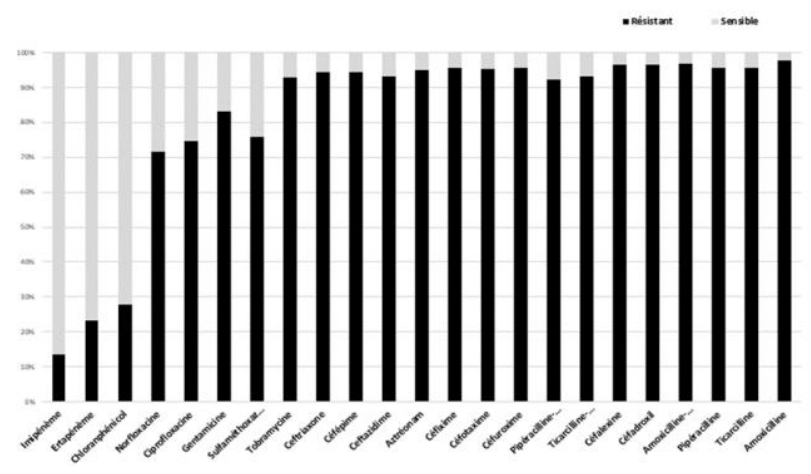

Fig 1. Resistance profile of Enterobacteriaceae

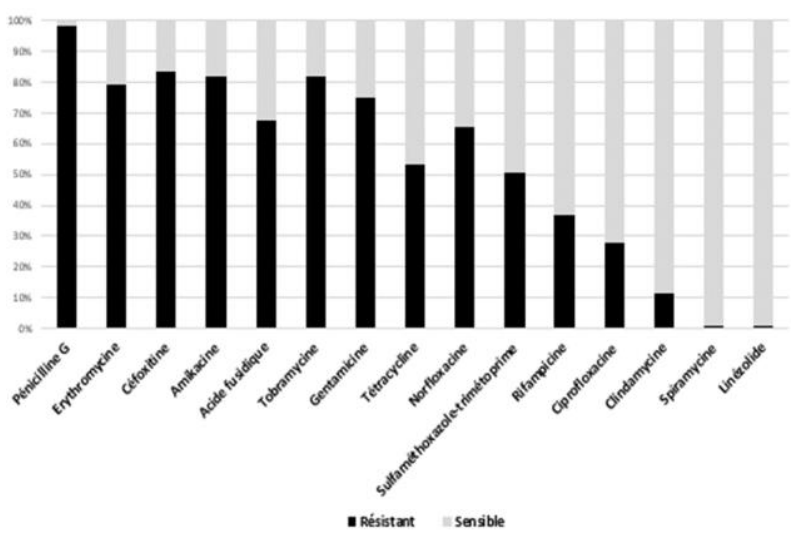

Fig 2. Staphylococcal resistance profile

\section{Discussion}

\section{Microbiological epidemiology}

SCNs are the major components of the skin flora, and thus can easily contaminate blood cultures. The same problem occurs with the rest of the skin flora (Bacillus spp, Corynebacterium, Stresptococus $\alpha$-hemolytic and Propionibacterium acnes...) [ 5]

Asserting the responsibility of these germs in a septic state remains a delicate exercise. In most cases, and especially in intensive care units, these are patients with catheters, and it is therefore probably reasonable to consider them as true pathogens in bacteremia with two positive blood cultures with the same strain, or even a single blood culture, while answering with the comment "a single blood culture taken, low predictive value. To be interpreted according to the clinic and the asepsis of the sample".[5] For this reason, we have followed the

*Loubna Yacoubi : loubnayacoubi1993@gmail.com 
REMIC recommendations and all the bacteremias we have isolated are true.

In our work, CNS is the most isolated group of bacteria from positive blood cultures $(n=114 ; 41.45 \%)$. These results are in agreement with the data reported by a study in Turkey over a period of 7 years [6] and a study in Iran over a period of one year [7].

BGN bacteremia remains largely dominated by Klebsiella pneumoniae as found by national and international authors with variable rates [8-11]. in our study $20.36 \%(n=56)$ of Klebsiella pneumonia

Abaumannii is the species that occupies the third position after klebseilla pneumoniae with a rate of $10.9 \%$ $(n=30)$. This frequency of isolation is discordant with the study of Hilal Ozkan reporting a rate of isolation of $3.31 \%[6]$

\section{Resistance profile}

The susceptibility of germs to antibiotics depends on the epidemiological situation of each hospital and the microbial ecology of each facility. The severity of neonatal infections in the intensive care setting is largely due to the multidrug resistant of the bacteria involved. The selection pressure linked to the antibiotic treatments administered and the existence of a genetic support in the environment allowing the selection of resistant strains are important factors in the evolution of antibiotic resistance [12].

SCN its profile was marked by a high resistance to meticillin, of the order of $100 \%, 80 \%$, respectively in Peru [13] and France [15], In our study, the rate reached $57.89 \%(n=66)$ of SCN strains

In our study, $96 \%$ of isolated Klebsiella pneumoniae strains were ESBL producers. This is in agreement with what is reported by Heloise Buys $86 \%$ [15].

In our work, $73 \%(n=22)$ of $A$. baumannii strains were resistant to imipenem. A higher rate was reported in the study of M. Lemsanni in Marrakech (90\%) [10] and a lower rate in a study conducted in Turkey by OZDEMIR at $53.3 \%$ [16].

\section{Conclusion}

The alarming increase of enterobacteriaceae isolates and their antibiotic resistance rates. This should encourage the reinforcement of hygiene measures within our university hospital.

\footnotetext{
*Loubna Yacoubi : loubnayacoubi1993@gmail.com
}

It is therefore necessary to have a collaboration between clinicians and biologists to identify this problem and limit the emergence of new resistant strains.

\section{References}

1. $\mathrm{Br}$ Med J Neonatal bacteraemia: diagnosis and management. British Medical Journal. (1979 Dec); 2 (6202):1385-1386.

2. Richard A. Polin and the COMMITTEE ON FETUS AND NEWBORN Management of Neonates With Suspected or Proven Early-Onset Bacterial Sepsis Pediatrics (May 2012),129 (5 )1006-1015;

3. Joshi SG, Ghole VS, Niphadkar KB. Neonatal gramnegative bacteremia. Indian J Pediatr. ( 2000 Jan ) ;67(1):27-32.

4. Recommandations du CASFM / EUCAST 2015 v.1.0. Comité de l'Antibiogramme de la Société Française de Microbiologie (SFM) - European Committee on Antimicrobial Susceptibility Testing (EUCAST).

5. Société Française de Microbiologie . Bactériémies et fongémies - hémocultures. Rémic : Référentiel en microbiologie $2015: 137-152 \mathrm{p}$

6. Hilal Ozkan,1 Merih Cetinkaya,1 Nilgün Koksal,1 Solmaz Celebi2 and Mustafa Hacımustafaoglu2 Culture-proven neonatal sepsis in preterm infants in a neonatal intensive care unit over a 7 year period: Coagulase-negative Staphylococcus as the predominant pathogen Pediatrics International (2014 ) 56, 60-66

7. Mohammadi P, Kalantar E, Bahmani N, Fatemi A, Naseri N, Ghotbi N, Naseri MH. Neonatal bacteriemia isolates and their antibiotic resistance pattern in neonatal insensitive care unit (NICU) at Beasat Hospital, Sanandaj, Iran. Acta Med Iran. ( 2014 ) ; 52(5):337-40.

8. N. Ben Jaballah, A. Bouziri, W. Kchaou, A. Hamdi, K. Mnif, S. Belhadj, A. Khaldi, K. Kazdaghli. Epidémiologie des infections bactériennes nosocomiales dans une unité de réanimation néonatale et pédiatrique tunisienne. Médecine et maladies infectieuses 36 ; (2006) ; 379-385.

9. M. Chemsi a, I. Chahid a, M. Lehlimi a, O. Aalloula b, K. Zerouali c, A. Habzi a, S. Benomar a. Incidence des infections bacteriennes nosocomiales. Hôpital d'enfants Abderrahim Harouchi, CHU Ibn Rochd, Casablanca, Maroc. Journal de pédiatrie et de puériculture (2013) ; 26, 11-18.

10. F. M. R. Maoulainine, N-S. Elidrissi, G. Chkil, F. Abba, N. Soraa, L. Chabaa, M. Amine, A. Aboussad. Epidémiologie de l'infection nosocomiale bactérienne dans un service de réanimation néonatale marocain. Archives de pédiatrie (2014) . ARCPED-3710; 1-6. 
11. Josette Raymond, MD ; Yannick Aujard, MD ; the European Study Group. Nosocomial infections in pediatric patients:aeuropean, multicenter prospective study. Vol. 21, No. 4 ; (2000)

12. Nicolas M.M, Espinasse F. Evolution de la flore responsable des infections nosocomiales. In : infection nosocomiale et résistance aux antibiotiques : évolution et tendances. Journées de l'hôpital Claude Bernard( 1993 ), Paris, Arnette : 13-28.

13. Becerra MR, Tantaleán JA, Suárez VJ, Alvarado MC, Candela JL, Urcia FC. Epidemiologic surveillance of nosocomial infections in a Pediatric Intensive Care Unit of a developing country. BMC Pediatr ( 2010$) ; \mathbf{1 0}: 66$.

14. Shefali O, Joy E, Daniel R, Colin M, Simon N. Neonatal causes of death estimates for the early an late neonatale periods for 194 contries. Bull World Health Organ. ( 2015 Jan 1 ) ; 93(1) :19-28.

15. Buys, H., Muloiwa, R., Bamford, C. et al. Klebsiella pneumoniae bloodstream infections at a South African children's hospital 2006-2011, a crosssectional study. BMC Infect Dis 16, 570 (2016).

16. Ozdemir $\mathrm{H}$, Kendirli $\mathrm{T}$ et al. Nosocomial infections due to Acinetobacter baumannii in a pediatric intensive care unit in Turkey. Turk J Pediatr ( 2011 May-Jun ); 53(3) :255-60.

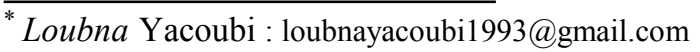

